\title{
Title: Palliative care nurses' recognition and assessment of patients with delirium symptoms: a qualitative study using critical incident technique
}

\author{
Authors: \\ Annmarie Hosie ${ }^{1}$ \\ Meera Agar ${ }^{2,3,4}$ \\ Elizabeth Lobb ${ }^{3,5,7}$ \\ Patricia M Davidson ${ }^{3,6}$ \\ Jane Phillips ${ }^{1,3,7}$
}

\section{Institutions:}

1. The University of Notre Dame, Sydney

School of Nursing

Cnr. Broadway and Abercrombie St

Broadway NSW 2007

Australia

2. Braeside Hospital

HammondCare

Department of Palliative Care

340 Prairie Vale Rd

Prairiewood NSW 2176

Australia

3. ImPaCCT: Improving Palliative Care through Clinical Trials

(New South Wales Palliative Care Clinical Trials Group)

South Western Sydney Clinical School

University of New South Wales

Faculty of Medicine NSW 2052 Australia

Sydney, NSW, Australia

4. Flinders University

Palliative and Supportive Services

Adelaide, South Australia, Australia

5. Calvary Health Care Sydney

Palliative Care Department

91-111 Rocky Point Rd.

Kogarah, NSW, Australia

6. University of Technology

School of Nursing, Midwifery and Health

Level 7, Building 10

Jones Street

Broadway. NSW 2007

Australia

7. Cunningham Centre for Palliative Care

Sacred Heart Hospice

St Vincent's Health Network

170 Darlinghurst St

Darlinghurst 2010 NSW

Australia

\section{Contact author:}

Annmarie Hosie

Phone number: 0417292077

Email: annmarie.hosie1@my.nd.edu.au 


\begin{abstract}
Background: Delirium is prevalent in palliative care inpatient settings and management is often challenging. Despite nurses' integral patient care role, little is known about palliative care nurses' capacity to recognise, assess and respond to patients' delirium symptoms.
\end{abstract}

Objective: To explore the experiences, views and practices of inpatient palliative care nurses in delirium recognition and assessment.

Settings and participants: 30 nurses from nine Australian specialist palliative care inpatient services.

Design and Methods: Critical incident technique (CIT) guided a series of semistructured interviews. Prior to interviews participants were given a vignette of a palliative care inpatient with an unrecognised hypoactive delirium, to prompt their recollection and recounting of a similar clinical incident. Clearly recalled and described incidents were analysed using thematic content analysis.

Findings: 20 of 30 participants recalled and described 28 relevant delirium incidents. Two themes and six sub-themes provide a general description of participants' experiences, views and practice in delirium recognition and assessment. Participants experience distress related to caring for patients with delirium and express compassion and empathy for delirious patients. Enhancing their delirium knowledge, strengthening collaborative multidisciplinary team relationships and better communication are important supports. Some participants, usually those in advance practice roles, describe more comprehensive assessment capabilities that incorporate clinical expertise with whole person awareness, yet systematic and structured delirium screening and assessment processes and application of the delirium diagnosis criteria 
are largely missing. Use of ambiguous terminology to describe delirium symptoms contributes to ineffective practice.

Conclusions: The findings of this study expands our understanding of how palliative care nurses' capacity to recognise and assess patients' delirium symptoms in the inpatient setting could be strengthened.

\section{Keywords:}

Assessment; Critical incident technique; Delirium; Nursing; Palliative care; Screening 


\section{Background}

Delirium is a complex neuropsychiatric syndrome that occurs frequently in palliative care inpatient populations, with delirium prevalence ranging from $13-42 \%$ on admission, 26-62\% during admission, and 58-88\% in the last weeks and hours of life (Hosie et al., 2013). The syndrome manifests as acute and fluctuating changes to patients' awareness, cognition and/or perception (American Psychiatric Association, 2000) causing distress for patients, both at the time of the delirium episode and later, when they recall their delirium experience (O' Malley et al., 2008, Breitbart et al., 2002). Patients with delirium exhibit several psychomotor symptoms, broadly classified into three subtypes: 1) hyperactive delirium - increased motor activity, agitation and heightened states of arousal; 2) hypoactive delirium - decreased motor activity, delayed response and drowsiness; and 3) mixed delirium - fluctuation between hyperactive and hypoactive states (Meagher, 2009, de Rooij et al., 2005, Gupta et al., 2008). In palliative care inpatient units, hypoactive delirium is most prevalent, associated with increased mortality and significantly under-recognised by clinicians (Fang et al., 2008, Lam et al., 2003, Spiller and Keen, 2006, Leonard et al., 2008). Despite its quieter presentation, patients experiencing this sub-type experience as much distress as those with hyperactive or mixed delirium (Breitbart et al., 2002). Delirium also adversely impacts on patients' relationships, function and ability to make decisions (Breitbart et al., 2002, Spiller and Keen, 2006) and leads to increased distress, anxiety and decision making for family members (Brajtman, 2003, Namba et al., 2007, Morita et al., 2007, Buss et al., 2007).

This serious, distressing syndrome has multiple potential causes - some iatrogenic (e.g. opioids, benzodiazepines, steroids and chemotherapeutic medications) (Agar and Lawlor, 2008) - and is potentially reversible (Lawlor et al., 2000, Leonard et al., 
2008), making early recognition of delirium symptoms and comprehensive assessment of the patient integral for targeted and optimal treatment and support (Canadian Coalition for Seniors' Mental Health, 2010, Registered Nurses Association of Ontario, 2003, Registered Nurses Association of Ontario, 2004). Delirium treatments and support ought be multi-faceted and individually tailored to: reverse the cause/s (e.g. administering antibiotics for infection or oxygen for hypoxia;

withdrawing or ceasing medication precipitants); ensure adequate nutrition, hydration, elimination and comfort and that hearing and vision aids are used; provide reassurance and information to the patient and family; promote a peaceful, safe ward environment; encourage the presence of patient's family; and/or administer psychotropic medications for severe delirium-related distress and agitation (Palliative Care Expert Group, 2010, Clinical Epidemiology and Health Service Evaluation Unit, 2006, Canadian Coalition for Seniors' Mental Health, 2010). If psychotropic medications are required, continued assessment of the patient's response and for presence of adverse effects, including delirium exacerbation, is essential (Canadian Coalition for Seniors' Mental Health, 2010, Meagher et al., 2013, Palliative Care Expert Group, 2010).

\section{Nurses' delirium knowledge, practice and experiences}

Nurses' intimate patient interactions over the 24 hour period makes them ideally placed to recognise early delirium symptoms, assess the patient and apply appropriate treatment and supportive interventions. Yet, nurses' capacity to effectively care for patients with delirium is limited by their: under-recognition of delirium (Steis and Fick, 2008, Voyer et al., 2012, Rice et al., 2011), knowledge and practice gaps (Fick et al., 2007, Hare et al., 2008a, Hare et al., 2008b) and distress and strain of caring for a patient with delirium (Breitbart et al., 2002, Belanger and Ducharme, 2011, 
Leventhal et al., 2013). Workplace culture and settings (Belanger and Ducharme, 2011, Agar et al., 2012) and nurses' personal values and philosophies (Mc Carthy, 2003) also shape their delirium knowledge, views and practices. Nurses who consider ageing as natural and not synonymous with disease are more likely to recognise delirium symptoms as a deviation from normal and consequently assess for potential causes, as do nurses with better knowledge of the patient and/or great role autonomy (Mc Carthy, 2003).

While two studies have explored palliative care nurses' perspectives of caring for patients with 'terminal delirium' (Brajtman, 2006) or delirium management generally (Agar et al., 2012), our study has intentionally focused on palliative care nurses' experiences, views and practices of delirium 'recognition' and 'assessment', primarily because these practice elements are pivotal points around which subsequent appropriate clinical decision-making and effective palliative care is implemented (World Health Organisation, 2002, Nursing and Midwifery Board of Australia, 2006).

\footnotetext{
Aims

To explore the experiences, views and practices of inpatient palliative care nurses in delirium recognition and assessment.
}

\section{Design and Methods}

\section{Critical incident technique}

Critical incident technique (CIT) guided semi-structured interviews and data analysis. CIT is a flexible set of principles applied to gather detailed information about how experts in a certain domain approach a procedure or significant situation and meanings they attach to this situation (Kemppainen, 2000, Flanagan, 1954, Keatinge, 2002). Defining features of CIT include: it focuses on determining facts, as opposed 
to generalisations, to find solutions to practical and real problems; uses either direct observation or obtains participants' clearly described memories of a specific incident; and these incidents, rather than participants, are the units of analysis. (Flanagan, 1954, Kemppainen, 2000, Butterfield et al., 2005). CIT identifies effective, ineffective and missing practices (Schluter et al., 2008), is useful for reflecting on professional practice (Hettlage and Steinlin, 2006) and has the advantage of allowing for brief interviews (Kemppainen, 2000). CIT was considered to be a feasible and nonthreatening method to achieve this study's aim (Butterfield et al., 2005), while also being a meaningful and powerful way to tap into clinical events that may have a lingering effect on nurses and their practice (Keatinge, 2002).

\section{Development and use of a vignette}

Vignettes are brief descriptions of clinical situations, used as an effective and economical means to explore or test clinician knowledge and/or decision-making (Veloski et al., 2005, Fick et al., 2007, Mc Crow et al., 2013). A vignette of a palliative care inpatient with unrecognised hypoactive delirium was developed and provided to participants shortly before their interview (Text Box 1). The vignette aimed to focus participants' attention onto delayed recognition of the symptoms of hypoactive delirium (the least recognised but most prevalent subtype of delirium in palliative care settings) (Spiller and Keen, 2006, Fang et al., 2008, Lam et al., 2003) and prompt their recall of a similar delirium incident from their clinical practice. It was anticipated that the vignette would be clinically relevant, familiar and accessible to all participants, regardless of their depth of delirium knowledge (Steis and Fick, 2008, Agar et al., 2012). After careful consideration, this approach was considered to be more appropriate than merely asking participants to recall and recount 'a patient experiencing delirium'. 
Vignette development was informed by the literature (Spiller and Keen, 2006, Meagher, 2009, Leonard et al., 2009). Validity, clarity and feasibility were undertaken by members of the research team [PD, MA, EL, JP], each of whom have clinical and research expertise in nursing, delirium and/or palliative care; and confirmed during pilot interviews with nurses $(n=4)$ with different levels of experience, from two palliative care units.

\section{Participants and settings}

Registered or enrolled nurses (New South Wales Government, 2011) working in clinical roles in Australian specialist palliative care inpatient settings, with at least three months experience in this setting and at least 12 months clinical experience overall, were eligible to participate. In Australia, a 'specialist palliative care service' is defined as: "a multi-disciplinary health care service whose substantive work is with patients who have a life limiting illness." (Palliative Care Australia, 2005, p.7).

\section{Recruitment and informed consent process}

Two discrete strategies were used to optimise recruitment and promote inclusion of a heterogeneous sample (Kemppainen, 2000), with invitations distributed via: 1) Specialist palliative care inpatient units; and 2) A nursing social media site (Hosie, 2013). Participants could choose to participate in a telephone or face-to-face interview (Sturges, 2004).

At participating units, nursing managers circulated the participant information and consent form to eligible nurses and notified them of interview times. Nurses who were interested in participating in a face-to-face interview met with the researcher $[\mathrm{AH}]$ at these times. Written consent was obtained after eligibility was confirmed, provision of information about the study and its voluntary nature, and questions answered. Nurses who expressed interest via the nursing social media site were 
emailed the participant information and consent form, and followed up within a week to ascertain their continued interest in participating [AH]. A scripted verbal consent was audiotaped prior to all telephone interviews, with participants forwarding their signed consent post interview.

\section{Ethical approval}

University and hospital ethical and governance approvals for this study were obtained prior to recruitment.

\section{Data collection}

The face-to-face interviews were conducted within a private room within each unit. The vignette was offered to participants between half to one hour before interviews, with most electing to read this in the private room just prior to interview commencement. The procedure for telephone interviews varied slightly: the vignette was emailed to participants a couple of hours prior to scheduled interviews, with $\mathrm{AH}$ unaware of the exact time of receipt. During telephone interviews, $\mathrm{AH}$ was located in a private office and participants in their workplace office or home. Consistent with the CIT, the interview was intentionally designed to be of around 20 minutes (Kemppainen, 2000). A question route (Text Box 2) was used for all interviews.

Interviews were audiotaped and conducted respectfully and supportively, so that participants felt safe to disclose incidents that were potentially difficult to share, due to the clinical situation or sub-optimal outcomes (Schluter et al., 2008). Participants were reassured that the interview was voluntary and its aim was not to 'test' their knowledge or review their individual performance, but to seek their insights. Interviews proceeded even when participants could not immediately recall a relevant incident, as some participants recalled and recounted an incident during their interview. When incidents were not generating any new behaviours, views, themes or 
sub-themes, indicating data saturation, a further five participants were recruited and interviewed to confirm data saturation (Flanagan, 1954, Kemppainen, 2000, Liamputtong and Ezzy, 2005).

\section{Reflexivity}

While not working as a colleague or manager of participants, the interviewer $[\mathrm{AH}]$ is an experienced palliative care nurse. This contextual knowledge required maintenance of objectivity, so open-ended and 'obvious' questions were asked during interviews and participants sometimes prompted to provide greater detail or explanation (Schluter et al., 2008, Flanagan, 1954). For example: “So when you say 'validation' what do you mean exactly by that?" During interviews, participants expressed varying views and practices in delirium recognition and assessment, at times surprising and differing to those of $\mathrm{AH}$, who was immersed in the delirium literature. This required $\mathrm{AH}$ to foster a heightened awareness of and appreciation for all participants' views and experiences, re-examine her own standpoint about nursing practice in delirium recognition and assessment, and investigate and reflect more deeply on what the nursing practice currently was, ought be and why nurses' views might differ (Jootun et al., 2009). Reflections were recorded, meditated upon and discussed with doctoral supervisors and fellow researchers.

\section{Analysis}

All interviews were transcribed verbatim. Field notes were completed immediately after each interview, noting relevant information not captured on tape, summarising key points and recording initial observations and insights (Schluter et al., 2008). From the interview transcripts, all incidents (the units of analysis) were collated into an electronic spread-sheet. In accordance with CIT only incidents with: i) an antecedent; ii) a clear and full description of the incident; and iii) an outcome, were 
included for data analysis (Flanagan, 1954). Any recollections that were vague, generalised or lacking in detail - suggesting either inaccurate recall or insufficient knowledge of the event (Flanagan, 1954) or not providing a clear outcome or information about effectiveness of actions (Bradbury-Jones and Tranter, 2008) - were omitted.

CIT has a distinct framework for data analysis that is consistent with other qualitative methodologies (Butterfield et al., 2005), with Flanagan (1954) providing broad recommendations for stages of data analysis: 1) Determining a frame of reference; 2) Formulating categories inductively; and 3) Determining level of specificity (i.e. dozens of specific behaviours) or generality (i.e. a few representative behaviours) to report the data (Flanagan, 1954, Butterfield et al., 2005). In our study, interview questions provided the frame of reference for initial recording of data (incidents) into an electronic spread-sheet $[\mathrm{AH}]$. Data analysis was an inductive process, using thematic content analysis (Glaser and Strauss, 1968, Schluter et al., 2011, BradburyJones and Tranter, 2008) and began during data collection. Transcripts, field notes and spread-sheets were read and re-read, promoting immersion in the data and close examination of individual incidents. Incidents and behaviours were then compared and contrasted: for example, what was occurring in incidents where there was more timely delirium recognition, assessment and intervention, compared to incidents where there was not? Theme and sub-theme development began [AH, JP]. Preliminary categories, themes and sub-themes were discussed with the researcher team [AH, JP, MA, LL, PD]; this analysis helped to refine the key themes and subthemes to more accurately reflect the data. Congruent with the exploratory aim, these themes and sub-themes represent a more general, rather than specific, description of incidents and participants' perspectives. 


\section{Findings}

There were 30 participants from nine specialist palliative care inpatient services in three Australian states (Table 1). Most were female $(n=29)$, worked in a major city location ( $n=28)$ and had over five years palliative care nursing experience $(n=20)$. Twelve participants worked in an advanced practice role, such as Clinical Nurse: Educator, Specialist, Consultant or Transitional Nurse Practitioner; and were more likely to have a relevant post-graduate qualification $(n=8)$ and longer length of palliative care experience. Twenty-five face-to-face and five telephone semistructured interviews were conducted in late 2012 - early 2013. Duration of interviews averaged 21 minutes (range 7-62 minutes), with variation of duration reflecting whether or not participants could recall and recount a relevant incident. Although 27 participants stated the vignette was familiar to them, only 20 provided a detailed description of one or two incidents involving recognition and assessment of acute changes to awareness, cognition and perception, generating 28 clearly described incidents that were included for analysis. All interviews took place during office hours, yet participants described incidents that occurred over the 24-hour period.

Thematic content analysis revealed the following two themes and six sub-themes:

1. The delirium experience:

i) Patients' delirium: causes, presentations and outcomes

ii) Concern for the patient and self

2. Nursing knowledge and practice in delirium recognition and assessment:

i) Challenges framing and naming observed changes

ii) Varying comprehensiveness of assessment 
iii) Inter-personal relationships and communication are valued

iv) Uncertainty and challenges promote desire for learning.

These themes and sub-themes are outlined in further detail below and summarised in Table 2.

\section{The delirium experience}

i) Patients' delirium: causes, presentations and outcomes

The incidents described included a broad range of symptoms and scenarios that were congruent with delirium phenomenology, (Meagher et al., 2007), causation (Lawlor et al., 2000, Gaudreau et al., 2005, Gaudreau et al., 2007) and reversibility in palliative care settings (Lawlor et al., 2000, Leonard et al., 2008). Participants attributed patient's delirium symptoms to a range of causes - often potentially modifiable $(n=12)$, such as: infection, hypoxia and medications (opiates, steroids, and an antipsychotic). Complete resolution of delirium occurred in almost half $(n=12)$ the incidents, while in three incidents the patients' symptoms persisted. Most participants labelled symptoms as 'delirium' $(n=14)$, while few $(n=2)$ identified the subtype.

All incidents involved acute changes to patients' awareness, cognition and/or perception. Participants' noted that delirium symptoms were sometimes mild and fleeting or on occasion developed quickly with escalating severity:

"He was alert and orientated on admission, but ...yesterday evening, he was starting to become a bit more unsettled and agitated. And then overnight he was quite paranoid and afraid that people were trying to keep him against his will. This morning... he is feeling that we're out to kill him and we're researching on him... and he's starting to use offensive language which is not in his normal personality." (P7) 
Emotional manifestations of delirium, such as anger, agitation or fear were also described:

"He was screaming at the top of his lungs... he was holding the buzzer, and he was saying that "That's a bomb" and he's angry with the nurses..." (P17)

Symptoms were sometimes initially attributed to patient's characteristics such as personality, but in retrospect recognised as having a physical cause which "... all made a lot of sense afterwards." (P16), and:

"Thinking back... he started with ... rambling conversation and not being able to focus, and the vagueness, and other signs that we were just attributing to the medications or he's just a bit strange ... I think we made excuses for a lot of the little behaviours earlier on.” (P27)

\section{ii) Concern for the patient and self}

Overwhelmingly participants expressed feelings of compassion, sadness, empathy or concern for patients experiencing delirium symptoms:

"It's distressing to see a patient be fearful of you... they're terminally ill, they might be in pain, and then you add this to their situation where they're lying in bed terrified, frightened and don't want you to come near them...I don't mind how it affects me but it upsets me to see how distressed they are." (P7)

Patients' distress recalling their delirium experience and their subsequent concerns about their behaviour or mental health meant that participants' concern for their patients and provision of support continued even when the delirium episode had resolved: 
"She knew that she wasn't like quite right: ..."I thought I was going crazy"...

"I know you did but you were perfectly safe, you know?" ... "I know you kept telling me... but I still felt a bit mad”. (P26)

Participants described feeling an onerous responsibility and isolation “...it's my duty of care if something happens to her, you know?" (P30), particularly on evening or night duty:

"I was only two years qualified at that stage and I was in charge of the ward that evening as well, and you don't have anyone to consult with ...” (P3) The incidents provoked feelings of helplessness, fear, frustration, and feeling out of their depth when managing the fluctuating changes associated with delirium

"You are wondering is it by talking to the patients, sitting with them and asking them what they are seeing and stuff like that, is that going to help? ... Sometimes you feel a bit isolated... a bit helpless... like: "Oh God, what am I going to do here?" (P3)

\section{Nursing knowledge and practice in delirium recognition and assessment}

\section{i) Challenges framing and naming observed changes}

Participants had difficulties framing the neurocognitive changes they had observed and linking them to a potential delirium diagnosis:

“Something about this patient, it's very unusual for her...we didn't know what's wrong with her...” (P30)

Symptoms were not explicitly integrated into a diagnostic or delirium framework:

"We were talking about... somebody who came across as a bit confused and a little bit vague, but the consensus with the team was that that was all 
personality rather than medication induced. I thought that was interesting, I'm like: "How do you figure out that?" (P9)

Participants often expressed feelings of surprise, puzzlement and frustration when describing the period before confirmation of a delirium diagnosis:

"The whole situation you were just feeling "Oh my gosh, what is it with him? How can we help him, why is he feeling like this? Is this part of his personality? ... He came in quiet and calm but is he showing his real self now?" ... Trying to work out what it was... you were a bit frustrated not being able to solve the problem there and then." (P12)

Some incidents involved patients seeing deceased family members or heavenly visions; participants were uncertain whether this was delirium or a spiritual or paranormal event:

"I had one patient that thought that they could see the gates, St Peter and the gates of Heaven. It was beautiful, she was in a great place, she was so happy and she said: "Can you see it?"...But is that delirium or is that a near death experience? Sometimes you don’t know." (P16)

When it was perceived that there was a non-physical cause for observed symptoms and/or alternative terminology such as terminal restlessness or agitation was adopted, this impeded understanding of delirium:

"What I've learnt is that we just don't pick it up. And that we often put everything into one bundle and we call it terminal agitation... I really believe that we really don't understand delirium at all." (P9) 
"It's hard to distinguish like delirium and then end-of-life terminal agitation... I don't know how to explain that one." (P30)

\section{ii) Varying comprehensiveness of assessment}

Comprehensiveness of patient assessment varied widely, from largely absent to broader assessments that were sensitive, holistic, inclusive of the patient, family and other team members and applied knowledge of potential causes of delirium symptoms (Nursing and Midwifery Board of Australia, 2006). When participants perceived that there was a spiritual or paranormal reasons for patients' report of hallucinations or illusions - such as a certain room on the ward being haunted - or when they attributed patients' perceptual disturbances to 'logical' misinterpretation of shapes or movement of objects in the room, they were less likely to undertake further assessment of the observed symptoms:

"She is seeing somebody in her room, but there is nobody there. First I thought she was confused and then I thought ... she was watching my reflection from the window... I didn't ask her detail because she (was) dozing off, so I thought “Oh...a dream, half dream"... but I didn't really pay attention or like telling doctor straight away" (P6)

Participants noted that nurses who labelled patients' presentations as 'terminal restlessness' were also less likely to undertake further assessment and needed prompting to do so:

"My (nursing colleague) was using the terminology (terminal restlessness)... And I said, "Have we done a PR? Have we done a bladder scan? Have we checked the urine? ... He's a culturally and linguistically diverse gentleman and maybe he's unable to communicate effectively"... The nursing staff got 
back to me - even though he'd been urinating he had a bladder of 1,000 mls. So they've put a catheter in." (P11)

In some incidents, a basic physiological assessment of the patient was undertaken before informing the doctor of the observed changes:

"The patient is confused and we did all the observations... temperature, and then blood pressure, and then respirations, oxygen saturations... initially I thought she was toxic to the opioids, so I checked the pupils...but she seems okay, she's not opioid toxic...(then) I told the doctor.” (P30)

Participants working in advanced practice roles tended to describe more comprehensive assessment that included family member insights, the patient's phase of illness, goals of care, temporal pattern of symptoms and potential medication causes:

"Well ...I think it all comes down to a really good and thorough assessment, ... knowing that person's story... non-medical and medical, speaking to the family... what was normal for her last week, what have we done since last week, where are we at with our disease process... all of those different things, how are we treating, what are we treating." (P26)

Although a small number of participants referred to cognition and delirium assessment tools such as the Mini-mental State Examination (MMSE) (Folstein et al., 1975) and the Confusion Assessment Method (CAM) (Inouye et al., 1990, Ryan et al., 2009), none described their application in their recalled incidents. Two participants stated their hospital's delirium policy gave them guidance on searching for potential physical causes of delirium or delirium symptoms (e.g. laboratory results, 
physiological measures, urinary retention). Otherwise, participants did not describe using systematic and structured delirium assessment processes.

\section{iii) Inter-personal relationships and communication are valued}

The most often described and perceived effective strategies for delirium recognition and assessment was development and fostering of relationships and communication between nurses, patients, family members and doctors. Team communication included reporting the symptoms to the doctor and/or the team leader, documenting what was happening for the patient and discussing possible causes and interventions. Participants reported that collaborative communication with doctors supported timely assessment of delirium causation:

"We sat down and we talked about the behaviours that had been happening over the last few days... Dr (Name) was saying, "Do you think it might be delirium...maybe we shouldn't be throwing more medication at this man. We have to find out what's going on," and it was the next day they start doing scans.” (P27)

Rapport and shared values between nurses and doctors were considered important:

"I then waited until the consultant came in... and spoke to him directly... He actually listened to me.... she ended up on IV antis (antibiotics) and reduction in her opioids and she returned to normal and she went home." (P11)

Prior knowledge of the person, through an existing nurse-patient relationship or actively seeking to know the person was described as a factor in recognising delirium symptoms: 
"He'd come into hospital and suddenly developed a lot of agitation and restlessness, which was abnormal for him, and confusion. I had met this man before outside hospital and he was of sound mind." (P10)

Not knowing the patient meant participants often struggled to make sense of symptoms, such as for this newly admitted patient who spoke little English:

"She would lie in her bed really quietly...tucked right under the covers and her eyes were just really wide open, but we couldn't verbally... work out what was wrong with her, but she always had this frightened look on her face and when her family came to visit ...they told us that ... she felt really scared because she was seeing someone in the room with her." (P16)

Proactive communication with families elicited further information: "I rang her daughter and spoke to her..." (P23). Effective communication further required a preparedness to have sensitive and profound conversations with patients about their delirium experience:

"Eventually came out that she was scared about her own death because to her (his) ... presence meant that her time was coming closer, she was apprehensive... so she would ask questions like, "How is that going to happen? Am I going to be in pain? Will I be here? Will I be at home? Who will find me?" (P26)

\section{iv) Uncertainty and challenges promote desire for learning}

Participants identified that gaps in their delirium knowledge had contributed to their uncertainty, puzzlement and delays in appropriate interventions. Delirium had been largely absent from palliative care education undertaken: 
"I've done the ABC of palliative care and ... advanced symptom management and I don't recall delirium ever coming along as being one of those things that we would look at if a patient was confused or agitated. It has never been brought up..." (P27)

For some participants, experience of uncertain and difficult delirium incidents had created 'tensions', prompting reflection and subsequent steps to improve their own delirium knowledge and to educate others. The following quote outlines how caring for a man experiencing severe delirium symptoms for several days (eventually determined to be precipitated by steroid medication) had impacted upon this participant's experiential learning and desire to teach other nurses:

"I'll always remember that now with this case. That was a good learning...We got the doctor to give us an in-service (after) that, to help us understand more ... as a CNS (Clinical Nurse Specialist) I'm probably going to ... look into it more and give education to other nursing staff... so they can be aware of that and in the future if they come across it, they'll know how to deal with it." (P12)

However, the delirium education participants had sought had not always met their own learning needs:

"I went to the delirium study day.... it was really good but I found it was very medical based, I think we need more our level.” (P03)

This participant believed debriefing at the unit level might promote better integration of delirium evidence into nurses' knowledge and practice: 
"We all know about evidence based practice, but how do we integrate that into the ward?... I think we need more opportunities to debrief and break things down... like: Where was that? Where did we miss it? What was the first trigger? You know, more opportunities to really educate ourselves.” (P09)

\section{Discussion}

\section{Knowledge}

These palliative care nurses had rich experiences of caring for patients with delirium symptoms; but despite this experience, there were varying delirium recognition and assessing capabilities. The findings from our study support Steis and Fick's' belief that nurses' delirium 'knowledge', 'recognition' and 'assessment' are distinct but inter-related concepts, and that nurse under-recognition of delirium as a syndrome - as opposed to delirium symptoms per se - is due to nurses' limited delirium diagnostic criteria knowledge (Steis and Fick, 2008). In our study, diagnostic criteria for delirium were not referred to during any of the incidents, with this absence contributing to nurses' puzzlement, worry and frustration continuing for a period of time (sometimes days) when they were not able to quickly make sense of what was happening for the patient, resolving only when it was determined - usually by the doctor - that delirium was the cause of the acute changes. Limited delirium knowledge and ability to apply the delirium diagnostic criteria to 'frame' delirium symptoms was similarly identified in another study exploring Australian palliative care, aged care, aged care psychiatry and oncology nurses' practice (Agar et al., 2012).

Although establishing any diagnosis is primarily a medical responsibility, nurses are required to develop understanding and expertise in recognising early signs and 
symptoms of prevalent syndromes and conditions and then proceed to comprehensive patient assessment, to effectively manage patients' care and communicate their findings to other members of the interdisciplinary team (Nursing and Midwifery Board of Australia, 2006, Registered Nurses Association of Ontario, 2003, Registered Nurses Association of Ontario, 2004). Delirium is so intrinsically linked to illness or frailty, where the need for nursing care is greatest, so nurses ought have a major assessment role. Yet the syndrome's diagnostic criteria is predominantly held within the realms of psychiatry (American Psychiatric Association, 2013, American Psychiatric Association, 2000) and medicine, meaning that nurses are literally 'two steps removed' from this principal knowledge source and have unintentionally been excluded from developing a shared understanding of delirium, delirium recognition capabilities and contributing to the diagnostic process. This may in part explain why within the discipline there is sub-optimal knowledge of delirium or understanding of the key nurse role in proactively recognising, assessing and managing this debilitating syndrome. To ensure all patients have access to exemplary care, all nurses must have equitable and timely access to evolving delirium knowledge and diagnostic criteria such as recently revised and amended (American Psychiatric Association, 2013) with translation and integration of this diagnostic criteria into everyday palliative care clinical practice and systems a critical first step towards developing nurses' delirium recognition and assessment capabilities (Registered Nurses Association of Ontario, 2004).

Reflective of gaps in nurses' delirium knowledge, failure to use correct delirium terminology has also been described as an "absence" within nursing delirium discourses (Kjorven et al., 2011, p.332). Similarly, our study found that applying ambiguous terms such as 'terminal restlessness/agitation', commonly used in 
palliative care, also made it difficult for nurses to conceptualise delirium, link their observations of patients' symptoms to a delirium framework and often led to inaccurate presumptions of dying, further limiting further assessment and intervention. There is an urgent need for the palliative care community to cease using this imprecise terminology, because of conceptual confusion, imprecision, potential to miss delirium and subsequently for missed opportunities to reverse the syndrome and inappropriate use of other interventions (Heyse-Moore, 2003, Hjermstad et al., 2004, Milisen et al., 2005).

Our study revealed that the challenging, emotional experiences of caring for delirious patients was the catalyst for some nurses to seek delirium knowledge and/or to teach others in order to better understand and manage future patients' delirium. However, nurses also identified deficits in availability and access to delirium education relevant to their palliative care practice. Nurses across care settings similarly report knowledge and education deficits (Brajtman, 2006, Flagg et al., 2010, Kjorven et al., 2011, p.332, Dahlke and Phinney, 2008), and highlights their need for more learning opportunities that are: linked to real patient scenarios, relevant to nursing and interdisciplinary palliative care practice, delivered at the unit or local level and evidence-based in content and delivery methods (Brajtman et al., 2008, Phillips et al., 2013). However, isolated education interventions to develop nurses' delirium knowledge are unlikely to be sufficient to optimise everyday nursing practice without additional systematic implementation of structured delirium recognition and assessment processes into local care settings (Registered Nurses Association of Ontario, 2003, Registered Nurses Association of Ontario, 2004, Balas et al., 2012). 


\section{Recognition}

'Recognition' is perception of sameness to something previously known (Macquarie

Dictionary, 2006). In our study, many participants gave clear and nuanced descriptions of multiple delirium symptoms, causes and outcomes, revealing they recognised acute neurocognitive changes had occurred for recalled patients, as well as their sequent impact; yet not all promptly recognised observed symptoms as delirium. Despite use of a hypoactive delirium vignette (albeit with perceptual disturbance), many incidents involved patients experiencing rapid change and overt behaviours or distress. As such, they may be considered a more 'critical incident' and recognised and remembered by nurses (Breitbart et al., 2002). Alternatively, including hallucinations in the vignette may have prompted recall of a range of delirium scenarios, as perceptual disturbances occur more commonly in mixed delirium (Meagher et al., 2011). Regardless, the incidents were fundamentally representative of the spectrum of delirium presentations that occur in inpatient palliative care settings (Meagher et al., 2007) and described included more key domains, particularly acute onset, than previously described by palliative care nurses (Agar et al., 2012). These richer details may be due to inclusion of the vignette; and applying the CIT, which gave participants an opportunity to give a detailed recounting of a relevant patient incident.

Although participants believed that knowing the patient well and communicating with them and their family supported recognition of delirium symptoms, nurses' bedside interactions with patients will not always lead to them detecting delirium (Mistarz et al., 2011). Aside from the challenge of recognising hypoactive delirium, not all patients with this syndrome will be known to nurses, able to communicate verbally or have family available, highlighting limitations of using unstructured delirium 
recognition approaches and sole reliance on patients' and family verbal capacity. Nurses are also less likely to document patients' delirium symptoms precisely if they do not use a structured screening process (Hare et al., 2008a, Steis and Fick, 2012).

In our study no nurse reported using a delirium screening tool, despite recommendations for use in high risk inpatient populations (Canadian Coalition for Seniors' Mental Health, 2010, Registered Nurses Association of Ontario, 2003, National Clinical Guideline Centre for Acute and Chronic Conditions, 2010), their availability in some workplaces, and routine daily symptom screening being a requirement in most Australian palliative care inpatient settings (Eagar, 2010, Aoun, 2011). This unstructured approach to delirium recognition and screening not only contributes to palliative care nurses' uncertainty, worry and puzzlement about observed symptoms but also delays the commencement of appropriate intervention for patients to reverse the delirium and/or reduce its negative impact. It is evident that there is great scope to increase early recognition of delirium through routine screening by nurses, to ensure delirium is immediately considered as a possibility when acute symptoms first occur and promote ongoing assessment and timely communication of observed changes to team members.

\section{Assessment}

Nursing assessment is an evidence-based, comprehensive, systematic and structured process that applies knowledge, incorporates patient data from a variety of sources, considers the patient holistically, is conducted sensitively and supportively and confirms findings with the patient and health care team (Nursing and Midwifery Board of Australia, 2006). Applying this definition, it is clear that delirium assessment practice varied considerably, with several examples provided of inadequate or absent assessment. Comprehensive assessment of the patient with 
delirium symptoms ought include investigation of physiological status, effects of medications, contributing environmental factors and support and information needs of the patient and their family (Registered Nurses Association of Ontario, 2004). Attribution of spiritual or paranormal causes for perceptual disturbances; and/or conceptualisation of delirium symptoms as terminal restlessness/agitation each contributed to nurses failing to conduct this necessary level of patient assessment, underscoring the importance of developing nurses' knowledge of delirium diagnostic criteria, prevalence and negative impact on patients, so that delirium assessment and timely intervention is viewed as a palliative care nursing priority. It is not surprising that advance practice nurses, compared to bedside nurses, more often described elements of comprehensive assessment, as this likely reflects their more autonomous roles, longer duration of experience and attainment of post-graduate qualifications. However, in our study no nurses described using a risk assessment to identify predisposing and precipitating delirium factors (Lawlor et al., 2000, Canadian Coalition for Seniors' Mental Health, 2010, National Clinical Guideline Centre for Acute and Chronic Conditions, 2010), or undertaking a baseline cognitive assessment using a validated tool routinely for all new admissions. In almost all incidents the use of structured guidance and a systematic process for the assessment of the patient with delirium was not described. Considering both the complexity of delirium and requirement for nursing assessment to be comprehensive, systematic and structured, this is a clear gap in palliative care nursing practice.

\section{Communication}

Nurses in our study perceived the most effective and valued practices in delirium symptom recognition and assessment were knowledge of the patient, collaborative team communication and inter-personal relationships with patients, families and 
colleagues, particularly doctors. Proactive communication combined with an empathetic approach is valued by patients and families, who desire and are reassured by provision of delirium information and a calm, warm and respectful approach that promotes patient dignity (Brajtman, 2003, Namba et al., 2007, Morita et al., 2007, Greaves et al., 2008). Further, effective team collaboration, communication and functioning is known to improve processes and outcomes of care, particularly in palliative, chronically ill and frail populations (Tieman, 2007, Abernethy et al., 2013) and is pivotal to team members' health and morale (Palliative Care Expert Group, 2010). However, nurses frequently report feeling ignored or not heard when reporting their delirium observations to doctors (Steis and Fick, 2008, Kjorven et al., 2011, p.332, Al-Qadheeb et al., 2013), potentially delaying intervention for the patient. This speaks to the need to design delirium communication strategies to strengthen interdisciplinary collaboration, mutual understanding, respect and effectiveness of care delivery (Vasilevskis et al., 2010).

\section{The experience of nursing delirious patients}

Across care setting, nurses universally feel incomprehension and discomfort when patients are delirious (Belanger and Ducharme, 2011). Similar to other studies exploring palliative care nurses' delirium experiences, this study has confirmed that nurses working in the palliative care setting experience distress when caring for patients with delirium (Brajtman, 2006, Breitbart et al., 2002, Agar et al., 2012). In our study, the predominant feelings nurses' expressed were compassion, concern and empathy combined with worry, frustration, fear, puzzlement, isolation, burden of responsibility and uncertainty: both about what might be happening to the patient and the best way to intervene. These findings reinforce the need for nurses to add structured delirium care processes into their daily practice and build their delirium 
recognition, assessment and management capabilities. Through development of delirium practice and knowledge, some of palliative care nurses' own professional support needs may be addressed, and their compassionate desire to help delirious patients better achieved.

\section{Strengths, limitations and challenges}

Like all qualitative studies these findings have limitations of transferability, as while the sample consists of nurses with varying roles and from several Australian palliative care units and different geographical locations, these nurses self-selected to participate and it is possible their experience reflects the views of nurses most interested in delirium. The strengths of this study include application of CIT, which allowed for brief, focused interviews and identifies effective, ineffective and missing practice, making it a feasible method to obtain nurses' perspectives and explore their professional delirium practice. While there were only a small amount of incidents compared to other CIT studies, this is likely related to the exploratory nature of the study combined with the focus on a narrow aspect of delirium care, namely inpatient palliative care nurses' recognition and assessment practices. Similar to previous CIT nursing studies (Bradbury-Jones and Tranter, 2008), a third of participants did not recount a specific clinical incident, despite the use of a vignette to prompt recall. Difficulty recalling a relevant incident may relate to under-recognition of delirium symptoms; alternatively, participants may not have been given sufficient time for recollection. As CIT also relies on participants' capacity to accurately recall and express past events and actions, the recounted incidents may not fully reflect the event or the extent of participants' actions. Adhering to the CIT's methods for data inclusion and analysis ensured rigor (Bradbury-Jones and Tranter, 2008, Butterfield et 
al., 2005), yet also resulted in exclusion of delirium insights of participants who could not recall an incident.

\section{Conclusion}

These findings expand our understanding of how nurses might actively engage in the building of better systems and clinical capacity, to better recognise and assess patients' with delirium symptoms in palliative care inpatient settings in the future.

\section{Implications for practice, education and research}

Given the prevalence and incidence of delirium in specialist palliative care setting (Hosie et al., 2013), a "high index of suspicion” by nurses is warranted (Le Grand, 2012, Registered Nurses Association of Ontario, 2003). It requires making delirium screening on and during admission routine practice, particularly when potentially delirium inducing interventions are introduced (Hosie et al., 2013, Rao et al., 2011). The extent of integration of systematic and structured processes for optimal delirium care within palliative care inpatient settings should be quantified. We need to further investigate whether implementation of a routine, structured delirium assessment process improves the capacity of nurses to recognise, assess and communicate patients' delirium symptoms (Detroyer et al., 2013); and importantly, which elements of practice change lead to better delirium outcomes and improved quality of life for palliative care patients and families (Gagnon et al., 2012). Advance practice nurses have an important role in defining, teaching and diffusing exemplar delirium practice within palliative care units. Further investigation of palliative care nurse delirium learning needs is required, as is subsequent development and testing of accessible delirium educational resources relevant to the context and team practice (Brajtman et al., 2008, Teodorczuk et al., 2013); for example, online delivery methods and their impact on knowledge, practice and patient outcomes (Phillips et al., 2013). Effective 
palliative care requires optimal collaboration and communication between team members, so interdisciplinary communication strategies - such as shared knowledge, language, tools and daily discussion (Brajtman et al., 2008, Balas et al., 2012, Vasilevskis et al., 2010) - to improve delirium recognition, assessment and intervention could be tested in the palliative care setting. For future practice and system interventions to improve delirium outcomes in palliative care populations, further knowledge of delirium epidemiology, contextual factors and patient, family and clinician perspectives of delirium care is needed. There is much work to be done to improve recognition, assessment and management of delirium in palliative care and nurses must be part of the solution.

Acknowledgements: The authors would like to acknowledge the time, support and insights contributed by palliative care nurses and their managers to this study.

Funding: An Australian Postgraduate Award from the Commonwealth Government of Australia supported Ms Hosie in this work. 
Table 1: Characteristics of the sample $(n=30)$

\begin{tabular}{|c|c|}
\hline Characteristic & Number \\
\hline \multicolumn{2}{|l|}{ Gender } \\
\hline Female & 29 \\
\hline Male & 1 \\
\hline \multicolumn{2}{|l|}{ Age } \\
\hline $21-30$ & 3 \\
\hline $31-40$ & 11 \\
\hline $41-50$ & 9 \\
\hline $51-60$ & 6 \\
\hline $61-70$ & 1 \\
\hline \multicolumn{2}{|l|}{ Position Title } \\
\hline Registered Nurse & 16 \\
\hline Clinical Nurse Specialist & 6 \\
\hline Enrolled Nurse & 2 \\
\hline Clinical Nurse Consultant & 2 \\
\hline Nursing Unit Manager & 2 \\
\hline Clinical Nurse Educator & 1 \\
\hline Nurse Practitioner Candidate & 1 \\
\hline \multicolumn{2}{|l|}{ Highest qualification } \\
\hline Certificate & 5 \\
\hline Diploma & 4 \\
\hline Bachelor & 9 \\
\hline Post graduate certificate & 9 \\
\hline Post graduate diploma & 7 \\
\hline \multicolumn{2}{|l|}{ Type of palliative care inpatient service } \\
\hline Direct care, mixed unit & 17 \\
\hline Direct care, palliative care patients only & 10 \\
\hline Consultative & 3 \\
\hline \multicolumn{2}{|l|}{ Geographical location of workplace $\star$} \\
\hline Major city & 28 \\
\hline Inner regional & 1 \\
\hline Outer regional & 1 \\
\hline Remote & 1 \\
\hline \multicolumn{2}{|l|}{ Years of nursing experience } \\
\hline $1-3$ years & 2 \\
\hline $3-5$ years & 5 \\
\hline $6-10$ years & 2 \\
\hline $11-15$ years & 6 \\
\hline $16-20$ years & 5 \\
\hline$>21$ years & 10 \\
\hline \multicolumn{2}{|l|}{ Years of palliative care experience } \\
\hline$<1$ year & 1 \\
\hline $1-3$ years & 5 \\
\hline $3-5$ years & 4 \\
\hline $6-10$ years & 9 \\
\hline $11-15$ years & 8 \\
\hline $16-20$ years & 1 \\
\hline$>21$ years & 2 \\
\hline
\end{tabular}

Totals more than 30 because one participant worked in more than one geographical area 


\section{Text Box 1: Vignette}

Mrs X is admitted to your palliative care unit on Monday. She is widowed, aged 81, lives alone and her diagnosis is advanced lung cancer. The reason for admission is for symptom management, as she has escalating pain. She has a son and daughter, but she is unaccompanied by any family or friends at admission. Medical and nursing admission processes are completed. Mrs X was independent with ADLs prior to admission. She shares a four-bed room with 3 other female patients.

Her opioid and adjuvant doses are increased after admission and by day 3 her pain appears to be improving.

Mrs $\mathrm{X}$ is a quiet, cooperative lady who displays no signs of agitation, but is noted to be a little vague in her verbal responses. She interacts only occasionally with the other patients in the room. She sleeps for intervals during the day, and is sometimes slow to rouse. Night staff report that she is awake for periods of time each night. When awake, she sits quietly and watches what is happening in the room.

Her son visits her each evening after he finishes work. On the evening of the 4th day of admission, he speaks to the nurse on duty and tells her that his mother has told him that she can see a dead man in the corner of the room, and that it has been there since she arrived on the ward. He also reports that his mother is not as clear in her speech and thinking as is usual for her.

The nurse speaks to Mrs X about this. Mrs X says she has been wondering why no one has talked about this man and that she was too frightened to report what she was seeing, in case people thought she was 'crazy'. She reveals that she finds the sight of the dead man very disturbing, and is worried she is 'losing her marbles'. She also reports she is finding it harder to concentrate and remember simple things. 


\section{Text box 2 - Interview route}

\section{Introduction to the interview}

"Thank you for agreeing to participate. The interview may take about 20 minutes. It will be audiotaped and I may also take some notes during the interview. Is that $\mathrm{OK}$ with you?

During the interview I will ask you some questions about your experience in nursing a palliative care patient who has acute changes in their awareness, thinking and perception, with the focus on how nurses recognise and assess these changes. The interview is not meant to be a test, we are mainly looking for insights into what nurses think are the most important things to do when caring for patients with these changes. You might find you feel a bit nervous, or as you recall your experiences it is possible this may bring up some feelings for you. It is OK to not answer all of the questions, or to ask for a break if you need it.

Have you had a chance to read the case study? Are you ready to start now?"

\section{Interview questions}

1. Does this case study reflect a situation you have observed or experienced recently in your own clinical practice?

2. Can you tell me about one particular patient situation in detail?

3. How did you feel about the situation?

4. What did you do?

5. In looking back at that situation, is there anything you would do differently?

6. Thinking about the future, do you have any suggestions for what we as nurses could do to better recognise the changes and manage the situation?

\section{Conclusion of the interview}

"Thank you for your time - I really appreciate your input. Do you have any further comments or questions? Remember, you can contact me by phone or email if you want to discuss the interview or study." 


\section{References}

Abernethy, A. P., Currow, D. C., Shelby-James, T., Rowett, D., May, F., Samsa, G. P., Hunt, R., Williams, H., Esterman, A. \& Phillips, P. A. 2013. Delivery strategies to optimize resource utilization and performance status for patients with advanced life-limiting illness: Results from the "palliative care trial" [ISRCTN 81117481]. Journal of Pain and Symptom Management, 45, 488505.

Agar, M., Draper, B., Phillips, P. A., Phillips, J., Collier, A., Harlum, J. \& Currow, D. 2012. Making decisions about delirium: A qualitative comparison of decision making between nurses working in palliative care, aged care, aged care psychiatry, and oncology. Palliative Medicine, 26, 887-96.

Agar, M. \& Lawlor, P. 2008. Delirium in cancer patients: A focus on treatmentinduced psychopathology. Current Opinion in Oncology, 20, 360-366.

Al-Qadheeb, N. S., Hoffmeister, J., Roberts, R., Shanahan, K., Garpestad, E. \& Devlin, J. W. 2013. Perceptions of nurses and physicians of their communication at night about intensive care patients' pain, agitation, and delirium. American Journal of Critical Care, 22, e49-61.

American Psychiatric Association 2000. DSM-IV-TR: Diagnostic and statistical manual of mental disorders Washington DC, American Psychiatric Association.

American Psychiatric Association 2013. Diagnostic and statistical manual of mental disorders, Fifth Edition (DSM-5), Arlington, VA, American Psychiatric Publisher.

Aoun, S. M. M., L. Kristjanson, L. J. Mc Conigley, R. 2011. Measuring symptom distress in palliative care: Psychometric properties of the symptom assessment scale (SAS). Journal of Palliative Medicine, 14, 315-321.

Balas, M. C., Vasilevskis, E. E., Burke, W. J., Boehm, L., Pun, B. T., Olsen, K. M., Peitz, G. J. \& Ely, E. W. 2012. Critical Care Nurses Role in Implementing the ABCDE Bundle Into Practice. Critical Care Nurse, 32, 35-47.

Belanger, L. \& Ducharme, F. 2011. Patients' and nurses' experiences of delirium: a review of qualitative studies. Nursing in critical care, 16, 303-315.

Bradbury-Jones, C. \& Tranter, S. 2008. Inconsistent use of the critical incident technique in nursing research. Journal of Advanced Nursing, 64, 399-407.

Brajtman, S. 2003. The impact on the family of terminal restlessness and its management. Palliative Medicine, 17, 454-460.

Brajtman, S., Hall, P., Weaver, L., Higuchi, K., Allard, P. \& Mullins, D. 2008. An interprofessional educational intervention on delirium for health care teams: Providing opportunities to enhance collaboration. Journal of Interprofessional Care, 22, 658-660.

Brajtman, S. H., K. \& Mc Pherson, C. 2006. Caring for patients with terminal delirium: palliative care unit and home care nurses' experiences. International Journal of Palliative Nursing, 12, 150-6.

Breitbart, W., Gibson, C. \& Tremblay, A. 2002. The delirium experience: delirium recall and delirium-related distress in hospitalized patients with cancer, their spouses/caregivers, and their nurses. Psychosomatics, 43, 183-94.

Buss, M. K., Vanderwerker, L. C., Inouye, S. K., Zhang, B., Block, S. D. \& Prigerson, H. G. 2007. Associations between caregiver-perceived delirium in patients with cancer and generalized anxiety in their caregivers. Journal of Palliative Medicine, 10, 1083-92. 
Butterfield, L. D., Borgen, W. A., Amundson, N. E. \& Maglio, A.-S. T. 2005. Fifty years of the critical incident technique: 1954-2004 and beyond. Qualitative Research, 5, 475-497.

Canadian Coalition for Seniors' Mental Health 2010. Guideline on the Assessment and Treatment of Delirium in Older Adults at the End of Life. Toronto: Canadian Coalition for Seniors' Mental Health (CCSMH).

Clinical Epidemiology and Health Service Evaluation Unit, M. H. 2006. Clinical Practice Guidelines for the Management of Delirium in Older People. Melbourne: Australian Health Ministers' Advisory Council (AHMAC).

Dahlke, S. \& Phinney, A. 2008. Caring for hospitalized older adults at risk for delirium: The silent, unspoken piece of nursing practice. Journal of Gerontological Nursing, 34, 41-47.

De Rooij, S. E., Schuurmans, M. J., Van Der Mast, R. C. \& Levi, M. 2005. Clinical subtypes of delirium and their relevance for daily clinical practice: a systematic review. International Journal of Geriatric Psychiatry, 20, 609-15.

Detroyer, E., Clement, P. M., Baeten, N., Pennemans, M., Decruyenaere, M., Vandenberghe, J., Menten, J., Joosten, E. \& Milisen, K. 2013. Detection of delirium in palliative care unit patients: A prospective descriptive study of the Delirium Observation Screening Scale administered by bedside nurses. Palliative Medicine.

Eagar, K. 2010. The Australian Palliative Care Outcomes Collaboration (PCOC) Measuring the quality and outcomes of palliative care on a routine basis. Australian Health Review, 34, 186-192.

Fang, C. K., Chen, H. W., Liu, S. I., Lin, C. J., Tsai, L. Y. \& Lai, Y. L. 2008. Prevalence, detection and treatment of delirium in terminal cancer inpatients: A prospective survey. Japanese Journal of Clinical Oncology, 38, 56-63.

Fick, D. M., Hodo, D. M., Lawrence, F. \& Inouye, S. K. 2007. Recognizing delirium superimposed on dementia: Assessing nurses' knowledge using case vignettes. Journal of Gerontological Nursing, 33, 40-47.

Flagg, B., Cox, L., Mcdowell, S., Mwose, J. M. \& Buelow, J. M. 2010. Nursing identification of Delirium. Clinical Nurse Specialist, 24, 260-266.

Flanagan, J. C. 1954. The Critical Incident Technique. Psychological Bulletin, 51.

Folstein, M. F., Folstein, S. E. \& Mc Hugh, P. R. 1975. 'Mini-Mental State'. A practical method for grading the cognitive state of patients for the clinician. Journal of Psychiatric Research 12, 189-198.

Gagnon, P., Allard, P., Gagnon, B., Merette, C. \& Tardif, F. 2012. Delirium prevention in terminal cancer: Assessment of a multicomponent intervention. Psycho-Oncology, 21, 187-194.

Gaudreau, Gagnon, P., Harel, F., Roy, M. \& Tremblay, A. 2005. Psychoactive medications and risk of delirium in hospitalized cancer patients. Journal of Clinical Oncology: Official Journal Of The American Society Of Clinical Oncology, 23, 6712-6718.

Gaudreau, Gagnon, P., Roy, M., Harel, F. \& Tremblay, A. 2007. Opioid medications and longitudinal risk of delirium in hospitalized cancer patients. American Cancer Society [Online].

Glaser, B. \& Strauss, A. 1968. The Discovery of Grounded Theory, Chicago, IL., Aldine.

Greaves, J., Vojkovic, S., Nikoletti, S., White, K. \& Yuen, K. 2008. Family caregivers' perceptions and experiences of delirium in patients with advanced cancer. Australian Journal of Cancer Nursing, 9, 3-11. 
Gupta, N., De Jonghe, J., Schieveld, J., Leonard, M. \& Meagher, D. 2008. Delirium phenomenology: What can we learn from the symptoms of delirium? Journal of Psychosomatic Research, 65, 215-222.

Hare, M., Mc Gowan, S., Wynaden, D., Speed, G. \& Landsborough, I. 2008a. Nurses' descriptions of changes in cognitive function in the acute care setting. Australian Journal of Advanced Nursing, 26, 21-25.

Hare, M., Wynaden, D., Mc Gowan, S., Landsborough, I. \& Speed, G. 2008b. A questionnaire to determine nurses' knowledge of delirium and its risk factors. Contemporary Nurse, 29, 23-31.

Hettlage, R. \& Steinlin, M. 2006. The Critical Incident Technique in Knowledge Management-Related Contexts: A tool for exploration / planning, evaluation and empowerment / animation [Online]. Zurich: Ingenious Peoples Knowledge. Available: http://www.i-pk.ch/files/CriticalIncidentTechnique_in_KM.pdf.

Heyse-Moore, L. 2003. Terminal restlessness and sedation: A note of caution [4]. Palliative Medicine, 17, 469.

Hjermstad, M. J., Loge, J. H. \& Kaasa, S. 2004. Methods for assessment of cognitive failure and delirium in palliative care patients: implications for practice and research. Palliative Medicine, 18, 494-506.

Hosie, A. 2013. Palliative care research [Online]. NSW Nurses and Midwives' Association. Available: http://www.nurseuncut.com.au/palliative-careresearch/ [Accessed Feb 1st 2013.

Hosie, A., Davidson, P. M., Agar, M., Sanderson, C. R. \& Phillips, J. 2013. Delirium prevalence, incidence, and implications for screening in specialist palliative care inpatient settings: A systematic review. Palliative Medicine, 27, 486-498.

Inouye, S. K., Van Dyck, C. H., Alessi, C. A., Balkin, S., Siegal, A. P. \& Horwitz, R. I. 1990. Clarifying confusion: the confusion assessment method. A new method for detection of delirium. Annals of Internal Medicine, 113, 941-8.

Jootun, D., Mc Ghee, G. \& Marland, G. R. 2009. Reflexivity: promoting rigour in qualitative research. Nursing standard (Royal College of Nursing (Great Britain): 1987), 23, 42-46.

Keatinge, D. 2002. Versatlity and flexibility: Attributes of the Critical Incident Technique in nursing research. Nursing and Health Sciences, 4.

Kemppainen, J. K. 2000. The critical incident technique and nursing care quality research. Journal of Advanced Nursing, 32, 1264-1271.

Kjorven, M., Rush, K. \& Hole, R. 2011, p.332. A discursive exploration of the practices that shape and discipline nurses' responses to postoperative delirium. Nursing Inquiry, 18, 325-335.

Lam, P. T., Tse, C. Y. \& Lee, C. H. 2003. Delirium in a palliative care unit. Progress in palliative care, 13, 126-133.

Lawlor, P. G., Gagnon, B., Mancini, I. L., Pereira, J. L., Hanson, J., Suarez-Almazor, M. E. \& Bruera, E. D. 2000. Occurrence, causes, and outcome of delirium in patients with advanced cancer: a prospective study. Archives of Internal Medicine, 160, 786-94.

Le Grand, S. B. 2012. Delirium in palliative medicine: A review. Journal of Pain and Symptom Management, 44, 583-594.

Leonard, M., Raju, B., Conroy, M., Donnelly, S., Trzepacz, P. T., Saunders, J. \& Meagher, D. 2008. Reversibility of delirium in terminally ill patients and predictors of mortality. Palliative Medicine, 22, 848-854. 
Leonard, M., Spiller, J., Keen, J., Mac Lullich, A., Kamholtz, B. \& Meagher, D. 2009. Symptoms of depression and delirium assessed serially in palliative-care inpatients. Psychosomatics, 50, 506-514.

Leventhal, M., Zimmerman, N., Denhaerynck, K., Lanz, E., Habegger, J. P., Muller, E. \& Siebenrock, K. 2013. Stress experienced in caring for patients with delirium in a university orthopedic and trauma surgery center. The 8th Annual Meeting of the European Delirium Association. Leuven, Belgium: European Delirium Association.

Liamputtong, P. \& Ezzy, D. 2005. Qualitative Research Methods, South Melbourne, Oxford University Press.

Mc Carthy, M. C. 2003. Detecting acute confusion in older adults: Comparing clinical reasoning of nurses working in acute, long-term, and community health care environments. Research in Nursing \& Health, 26, 203-12.

Mc Crow, J., Beattie, E., Sullivan, K. \& Fick, D. M. 2013. Development and review of vignettes representing older people with cognitive impairment. Geriatric Nursing, 34, 128-137.

Meagher, D. 2009. Motor subtypes of delirium: Past, present and future. International Review of Psychiatry, 21, 59-73.

Meagher, D. J., Leonard, M., Donnelly, S., Conroy, M., Adamis, D. \& Trzepacz, P. T. 2011. A longitudinal study of motor subtypes in delirium: Relationship with other phenomenology, etiology, medication exposure and prognosis. Journal of Psychosomatic Research, 71, 395-403.

Meagher, D. J., Mc Loughlin, L., Leonard, M., Hannon, N., Dunne, C. \& O' Regan, N. 2013. What Do We Really Know About the Treatment of Delirium With Antipsychotics? Ten Key Issues for Delirium Pharmacotherapy. American Journal of Geriatric Psychiatry.

Meagher, D. J., Moran, M., Raju, B., Gibbons, D., Donnelly, S., Saunders, J. \& Trzepacz, P. T. 2007. Phenomenology of delirium: Assessment of 100 adult cases using standardised measures. British Journal of Psychiatry, 190, 135141.

Milisen, K., Lemiengre, J., Braes, T. \& Foreman, M. D. 2005. Multicomponent intervention strategies for managing delirium in hospitalized older people: Systematic review. Journal of Advanced Nursing, 52, 79-90.

Mistarz, R., Eliott, S., Whitfield, A. \& Ernest, D. 2011. Bedside nurse-patient interactions do not reliably detect delirium: An observational study. Australian Critical Care, 24, 126-132.

Morita, T., Akechi, T., Ikenaga, M., Inoue, S., Kohara, H., Matsubara, T., Matsuo, N., Namba, M., Shinjo, T., Tani, K. \& Uchitomi, Y. 2007. Terminal delirium: recommendations from bereaved families' experiences. Journal of Pain \& Symptom Management, 34, 579-589.

Namba, M., Morita, T., Imura, C., Kiyohara, E., Ishikawa, S. \& Hirai, K. 2007. Terminal delirium: Families' experience. Palliative Medicine, 21, 587-594.

National Clinical Guideline Centre for Acute and Chronic Conditions 2010. Delirium: diagnosis, prevention and management, NICE Clinical Guideline 103. London: National Institute for Health and Clinical Excellence.

New South Wales Government 2011. Public Health System Nurses' and Midwives' (State) Award. In: HEALTH, N. M. O. (ed.). Sydney.

Nursing and Midwifery Board of Australia 2006. National Competency Standards for the Registered Nurse. Melbourne: Nursing and Midwifery Board of Australia. 
O' Malley, G., Leonard, M., Meagher, D. \& O' Keeffe, S. T. 2008. The delirium experience: A review. Journal of Psychosomatic Research, 65, 223-228.

Palliative Care Australia 2005, p.7. A Guide to Palliative Care Service Development: A population based approach. Deakin West: Palliative Care Australia.

Palliative Care Expert Group 2010. Therapeutic Guidelines: palliative care, Melbourne, Therapeutic Guidelines Limited.

Phillips, J. L., Shaw, T., Heneka, N., Hickman, L. \& Lam, L. 2013. The impact of a novel, online learning module on specialist palliative care nurses ${ }^{\text {ee }}$ pain assessment knowledge and practices European Palliative Care Association Conference. Prague, Czech Republic.

Rao, S., Ferris, F. D. \& Irwin, S. A. 2011. Ease of screening for depression and delirium in patients enrolled in inpatient hospice care. Journal of Palliative Medicine, 14, 275-279.

Registered Nurses Association of Ontario 2003. Screening for Delirium, Dementia and Depression in Older Adults (With revised 2010 supplement). Toronto: Registered Nurses Association of Ontario.

Registered Nurses Association of Ontario 2004. Caregiving Strategies for Older Adults with Delirium, Dementia and Depression (With revised 2010 supplement). Toronto: Registered Nurses Association of Ontario.

Rice, K. L., Bennett, M., Gomez, M., Theall, K. P., Knight, M. \& Foreman, M. D. 2011. Nurses' Recognition of Delirium in the Hospitalized Older Adult. Clinical Nurse Specialist, 25, 299-311 10.1097/NUR.0b013e318234897b.

Ryan, K., Leonard, M., Guerin, S., Donnelly, S., Conroy, M. \& Meagher, D. 2009. Validation of the confusion assessment method in the palliative care setting. Palliative Medicine, 23, 40-45.

Schluter, J., Seaton, P. \& Chaboyer, W. 2008. Critical incident technique: A user's guide for nurse researchers. Journal of Advanced Nursing, 61, 107-114.

Schluter, J., Seaton, P. \& Chaboyer, W. 2011. Understanding nursing scope of practice: A qualitative study. International Journal of Nursing Studies, 48, 1211-1222.

Spiller, J. A. \& Keen, J. C. 2006. Hypoactive delirium: assessing the extent of the problem for inpatient specialist palliative care. Palliative Medicine, 20, 17-23.

Steis, M. R. \& Fick, D. 2012. Delirium Superimposed on Dementia: Accuracy of Nurse Documentation Journal of Gerontological Nursing 38, 32-42.

Steis, M. R. \& Fick, D. M. 2008. Are nurses recognizing delirium? A systematic review. Journal of Gerontological Nursing, 34, 40-49.

Sturges, J. H., Kj. 2004. Comparing telephone and face-to-face qualitative interviewing: a research note. Qualitative Research, 4, 107-118.

Teodorczuk, A., Mukaetova-Ladinska, E., Corbett, S. \& Welfare, M. 2013. Reconceptualizing models of delirium education: findings of a Grounded Theory study. International Psychogeriatrics, 25, 645-55.

Tieman, J. 2007. Integration, coordination and multidisciplinary care: What can these approaches offer to Australian primary health care? Australian journal of primary health, 13, 56-65.

Vasilevskis, E. E., Ely, E. W., Speroff, T., Pun, B. T., Boehm, L. \& Dittus, R. S. 2010. Reducing iatrogenic risks: ICU-acquired delirium and weakness-crossing the quality chasm. Chest, 138, 1224-33.

Veloski, J., Tai, S., Evans, A. S. \& Nash, D. B. 2005. Clinical Vignette-Based Surveys: A Tool for Assessing Physician Practice Variation. American Journal of Medical Quality, 20, 151-157. 
Voyer, P., Richard, S., Mc Cusker, J., Cole, M. G., Monette, J., Champoux, N., Ciampi, A. \& Belzile, E. 2012. Detection of Delirium and Its Symptoms by Nurses Working in a Long Term Care Facility. Journal of the American Medical Directors Association, 13, 264-271.

World Health Organisation 2002. WHO Definition of Palliative Care. World Health Organisation. 